\title{
Strategies for the Electro-bioremediation of Oxyfluorfen Polluted Soils
}

\author{
Silvia Barba ${ }^{a}$, José Villaseñor ${ }^{\text {a,* }}$, Pablo Cañizares ${ }^{b}$ and Manuel A. Rodrigo ${ }^{b}$ \\ ${ }^{a}$ Department of Chemical Engineering, Institute of Chemical \& Environmental \\ Technologies, University of Castilla-La Mancha, Campus Universitario s/n, 13071
}

Ciudad Real, Spain

${ }^{b}$ Department of Chemical Engineering, Faculty of Chemical Sciences \& Technologies, University of Castilla-La Mancha, Campus Universitario s/n, 13071 Ciudad Real, Spain

* Corresponding author: Jose.villasenor@uclm.es

\begin{abstract}
This work compares three strategies for the in-situ remediation of oxyfluorfen polluted clay soil, based on the combination of biological processes and electrokinetics (EK): (i) EK-biostimulation, in which the action of microorganisms contained in soil is promoted without the use of biological permeable barriers, (ii) EK-bioaugmentation with fixed culture of microorganisms (BB1), which enhanced the natural bioremediation by including a microbial fixed biofilm reactor as permeable biobarrier, and (iii) EKbioaugmentation with suspended culture of microorganisms (BB2), which improves the natural remediation by including a mixture of soil and a microbial suspension as permeable biobarrier. To do this, two-week batch electro-bioremediation tests were carried out using bench scale set-ups. In each case, a constant electric field $\left(1 \mathrm{~V} \mathrm{~cm}^{-1}\right)$ was applied, and electrode polarity was reversed periodically, every $12 \mathrm{~h}$. In addition, a microbial consortium acclimated to oxyfluorfen biodegradation was used. The best oxyfluorfen removal efficiency (15\%) was obtained using the option (i). Permeable
\end{abstract}


biobarriers allowed the introduction of microorganism and nutrients into the soil, and the biological activity reached the whole soil positions. However, the insertion of these biobarriers caused an excessive decrease in the electro-osmotic flow (approximately $80 \%$ ) which, in turn reduced the mobilization of the pollutants. Despite of that, results show that permeable reactive biobarriers may result in a successful alternative for insitu EK-bioaugmentation, although higher retention times would be needed to obtain higher removal efficiencies.

\section{Keywords}

Biobarrier; Bioremediation; Electrokinetics; Oxyfluorfen; Polluted soil.

\section{Introduction}

Organochlorinated pesticides and herbicides are commonly used in agricultural activity for pest and weed growth control. Consequently, soil and groundwater pollution episodes often appear, causing severe environmental problems. The World Health Organization estimates that such substances are responsible to poison 2-3 million people and cause between 20000 and 200000 accidental deaths each year, primarily in developing countries [1]. Owing to their persistence in the environment, the removal of such chemicals from water and soils is necessary, because living organisms may be affected by the hazard that they entail. In addition, water reservoirs for human consumption could be affected. One of the most effective and widely used herbicides is oxyfluorfen, a non-polar diphenyl-ether herbicide, characterized by its very low water solubility $\left(0.116 \mathrm{mg} \mathrm{L}^{-1}\right)$ and low vapour pressure $\left(0.026 \mathrm{mPa}\right.$ at $\left.25^{\circ} \mathrm{C}\right)$ [2]. 
Because of the abovementioned soil pollution problems, its decontamination becomes necessary. There are different remediation alternatives for soils polluted with pesticides and herbicides [3]. One of the technologies is the electrokinetic treatment (EK), which is especially recommended for the in-situ treatment of low permeability soils. It consists of the application of an electric field among appropriately distributed electrodes inserted directly into soil or placed inside electrolyte wells. When an electric field is applied among those electrodes, several transport processes may occur (such as electro-osmosis, electromigration and electrophoresis) that move pollutants and other compounds such as surfactants, inorganic nutrients or even microorganisms [4]. The main objective of the electrokinetic remediation is to move the pollutants towards the nearness of the electrodes (or to the electrolyte wells), in order to carry out subsequently an external pollutant degradation. However, there are some possibilities to enhance the efficiency of the electrokinetic method by its combination with other types of technologies [5]. One of them is the combination with bioremediation, resulting in a technology so-called as "soil electro-bioremediation" [6].

Gill et al. [7] reviewed the different possibilities, processes and applications of the electrokinetic and biological combined technology for the remediation of organic contaminants. One option is the EK-bioattenuation. It improves the slow natural bioattenuation (a low impact and cost effective bioremediation technique which can be applied in the treatment of biodegradable pollutants) with the EK phenomena, which promote the mixing of contaminants, nutrients, electron acceptors and autochthonous microorganisms. A second option is EK-biostimulation, similar to bioattenuation, but in which biodegradation is accelerated by adding nutrients $(\mathrm{N}, \mathrm{P})$ and/or oxygen or other electron acceptors, improving redox conditions and increasing bioavailability. Finally, a third option is EK-bioaugmentation, which consists of the addition of microorganism 
and nutrients into the soil. One bioaugmentation option is to include permeable biological barriers into the polluted soil.

Generally, a permeable reactive barrier (PRB) is an artificial reactive material that is placed in the soil in the direction of groundwater flow, so that the PRB intercepts the pollution plume and retains or eliminates the pollutants [8]. Particularly, PRBs based on biological degradation are called as biological permeable reactive barriers (Bio-PRB) or biobarriers. A biobarrier can be a biofilm bioreactor (that is a porous fixed bed with a microbial culture attached on its surface) or just a wet mixture of soil and activated sludge [9]. Groundwater flow can be produced by conventional pumping methods and alternatively, by EK in low permeable soils. Although the number of publications on this topic is increasing rapidly, the combination of EK methods and PRB treatments is relatively recent and much work is still to be done in the next years for the complete understanding and optimization of the processes.

The electro-bioremediation technology has been previously tested by the authors of the present work, and different alternatives of combining bioremediation and electrokinetics were studied to remediate hydrocarbon-polluted soils [10]. The aim of the present work is to study such different electro-bioremediation strategies for the remediation of a oxyfluorfen polluted soil, through batch experiments using electrokinetics with periodic polarity reversal in three situations: (i) a EK-biostimulation alternative that consists of the application of electrokinetics to the polluted soil that already contains an oxyfluorfen-degrading microbial culture, (ii) a EK-bioaugmentation alternative that consists of the application of the microbial culture as a fixed biofilm reactor as permeable biobarrier, and (iii) a second EK-bioaugmentation alternative that consists of the application of the microbial culture as a mixture of soil and a suspension of microorganism as permeable biobarrier. Thus, the present work aims to evaluate 
different proposals for the improvement of in situ techniques for organochlorines polluted soils remediation. Oxyfluorfen is a persistent herbicide and biodegradation studies are scarce. Under the author's knowledge, no previous research (exception of previous works in our research group) has been found about electro-bioremediation of oxyfluorfen-polluted soil.

\section{Materials and methods}

The experimental installation as well as the experimental, sampling and analytical procedure, have been described elsewhere in previous research performed by the same authors and related to the same research programme $[9,10]$. The following subsections only describe the important details required for a good understanding of this work. Table 1 and 2 shows a summary of experiments carried out and parameters measured during the experiments respectively.

\subsection{Bench scale set-up}

The experiments were carried out in a bench scale electrokinetic cell (Figure 1a) that was made in transparent methacrylate and divided into five compartments. Oxyfluorfen polluted soil was introduced in the central compartment. The anodic and cathodic compartments were placed at both sides of the soil compartment, separated by nylon mesh (0.5 mm mesh size). They behaved as electrode wells, containing the electrolyte which is transported through the soil. Graphite plates $(10.0 \mathrm{~cm} \times 10.0 \mathrm{~cm} \times 1.0 \mathrm{~cm})$ were used as anode and cathode, and they were connected to the direct current power supply. In turn, each electrode compartment was connected to an external electroosmotic flow collection compartment. An electrolyte reservoir was connected to the collector compartment by means of a level control system. 


\subsection{Materials}

A clayey soil obtained from a quarry in Mora (Toledo, Spain) was used (12\% quartz, $23 \%$ kaolinite, $24 \%$ glauconite and $20 \%$ montmorillonite as the main components). Standards ASTM D2487 (Standard Practice for Classification of Soils for Engineering Purposes) and ASTM D4318 (Standard Test Methods for Liquid Limit, Plastic Limit, and Plasticity Index of Soils) were used. According to its granulometry, liquid limit and plasticity limit, the soil corresponded to a low plasticity clay. Fluoxil 24 EC (Cheminova Agro, Madrid), a commercial herbicide, was used to pollute the soil artificially. This product contains $24 \%$ oxyfluorfen, xylene $(<60 \%)$ and cyclohexanone $(<13 \%)$ as solvents, and calcium dodecylbencene sulfonate $(<4 \%)$ as surfactant, which is included to promote the correct spreading of oxyfluorfen in soils. Soil was vigorously mixed with a concentrated solution of Fluoxil 24 during several minutes in order to obtain artificially polluted soil containing $29 \mathrm{mg}$ oxyfluorfen per $\mathrm{kg}$ of wet soil and moisture below $25 \%$.

The oxyfluorfen-degrading microbial consortium was obtained from activated sludge of an oil refinery wastewater treatment plant, by a standard acclimation procedure described elsewhere [11]. The feeding solution for acclimation consisted of BushnellHass Broth (BHB) culture media (0.20 g Mg SO $4 \mathrm{~L}^{-1}, 0.02 \mathrm{~g} \mathrm{CaCl}_{2} \mathrm{~L}^{-1}, 1.00 \mathrm{~g} \mathrm{KH}_{2} \mathrm{PO}_{4}$ $\mathrm{L}^{-1}, 1.00 \mathrm{~g}\left(\mathrm{NH}_{4}\right)_{2} \mathrm{HPO}_{4} \mathrm{~L}^{-1}, 0.05 \mathrm{~g} \mathrm{FeCl}_{3} \mathrm{~L}^{-1}$ and $\left.1.00 \mathrm{~g} \mathrm{KNO}_{3} \mathrm{~L}^{-1}\right)$ and Fluoxil 24 (with a concentration of oxyfluorfen of $200 \mathrm{mg} \mathrm{L}^{-1}$ ) as the sole carbon source. Once the acclimation period was expected to be completed, the microbial culture performance was checked by several batch biodegradation tests as reported by Moliterni et al. [11]. Both the acclimation procedure and the batch oxyfluorfen biodegradation tests were conducted under no aerated conditions and using suitable amounts of nitrates (which acted as electron acceptor). Then, a MALDI TOF Mass Spectrometry AXIMA- 
Assurance equipment (Biotech technology, SHIMADZU, Germany) was used to identify the microbial species in the acclimated culture. The following species were identified in the acclimated microbial culture: Achromobacter denitrificans, Achromobacter xylosoxidans, Pseudomonas putida, Pseudomonas oryzihabitans and Brevibacterium casei.

The electrolyte used was a synthetic salt medium $\left(80.7 \mathrm{mg} \mathrm{L}^{-1} \mathrm{Na}_{2} \mathrm{SO}_{4}, 70.0 \mathrm{mg} \mathrm{L}^{-1}\right.$ $\mathrm{NaHCO}_{3}, 30.4 \mathrm{mg} \mathrm{L}^{-1} \mathrm{NaNO}_{3}$ ) and it was introduced into the electrode compartments in order to be distributed through the soil by the electrokinetic phenomena during the experiments. Such electrolyte provides electrical conductivity, it does not cause microbial toxicity and it has been successfully used in previously reported electrobioremediation works $[9,10]$. BHB medium was also used to supply nutrients to the soil or to the barriers, and an excess concentration of nutrients were supplemented in all experiments.

\subsection{Experimental procedure}

Three alternatives for the electro-bioremediation of the polluted soil were tested trough batch tests of 14 days length, all of them at room temperature using a constant electric field of 1.0 $\mathrm{V} \mathrm{cm}^{-1}(20.0 \mathrm{~V})$ and a periodic polarity reversal between the electrodes every $12 \mathrm{~h}$ (reversal frequency $\left.2 \mathrm{~d}^{-1}\right)$. The three alternatives are described as follows:

Case 1. EK-biostimulation through direct application of electrokinetics: An inoculum of the acclimated microbial consortium was grown in a batch reactor using BHB medium. The obtained culture was centrifuged and then suspended again in fresh BHB medium and it was added to the oxyfluorfen-polluted soil, which was moistened to a moisture content of approximately $25 \%$ adding also the inorganic salt medium. The soil was manually compacted into the central compartment and the direct electric current was connected. 
Case 2. EK-bioaugmentation through the inclusion of a fixed bed biofilm reactor as permeable biological barrier (BB1). The polluted soil was moistened to a moisture content of approximately $25 \%$, using the same synthetic salt medium as in the electrode compartments $\left(30.4 \mathrm{mg} \mathrm{L}^{-1}\right.$ of $\mathrm{NaNO}_{3}, 70.0 \mathrm{mg} \mathrm{L}^{-1}$ of $\mathrm{NaHCO}_{3}$ and $80.7 \mathrm{mg} \mathrm{L}^{-1}$ of $\mathrm{Na}_{2} \mathrm{SO}_{4}$ ) but no microbial consortium was added. Then, the soil was compacted identically as in the previous test. A part of the soil was then removed from the central position in the central compartment, and a $5 \mathrm{~cm}$ wide biological permeable reactive barrier (BB1) was loaded into this position and flooded with the BHB medium. The working principle of $\mathrm{BB} 1$ is the same as that of a conventional fixed bed biofilm reactor, and it was previously developed in the laboratory according to the procedure reported by Ramírez et al. [12] (Briefly: the fixed-bed biofilm bioreactor for oxyfluorfen-polluted wastewater treatment was developed using the acclimated culture, feeding BHB as the culture media and $200 \mathrm{mg} \mathrm{L}^{-1}$ of oxyfluorfen as the carbon source. The biofilm reactor worked in a weekly sequencing batch reactor operation mode using pumice stone as the supporting material with average diameter $3 \mathrm{~mm}$, and the hydraulic retention time was $150 \mathrm{~h}$. The reactor performance was checked under stationary conditions). The direct electric current was then connected.

Case 3. EK-bioaugmentation through the inclusion of a soil/microorganisms suspension mixture as permeable biological barrier (BB2). This option was identical to the previous one, and the only difference was that the central biobarrier (BB2) was easily prepared by just a mixture of clean clay soil and a suspension of the microbial culture in BHB medium. The soil/microorganisms mixture was located in the central position of the polluted soil. The direct electric current was then connected. Figure $1 \mathrm{~b}$ shows the bench scale set-up using both types of biobarrier.

Furthermore, two complementary reference experiments were carried out. In the first 
reference test, conditions were identical to the test 1 , although no electric current was applied to the soil (named as "No EK"). This test will help to know the possible evolution of oxyfluorfen biodegradation without the contribution of mobility caused by the electrokinetic phenomena. The second reference test was an abiotic EK reference test (named as "No Bio") and it was carried out by using the same electrokinetic conditions of the three experiments $\left(1.0 \mathrm{~V} \mathrm{~cm}^{-1}\right.$ and $2.0 \mathrm{~d}^{-1}$ polarity reversal frequency) but using no inoculated soil (no addition of acclimated oxyfluorfen removal microorganisms). This test will help to know the possible removal of oxyfluorfen by non-biologically assisted mechanisms, that is, EK removal to the external compartments, or evaporation.

\subsection{Sampling and analyses}

It is important to remark that, because of the polarity reversal every $12 \mathrm{~h}$, both electrode compartments were anode or cathode depending on the time, and thus electro-osmotic flow (EOF) was obtained in both electrode compartments as the polarity changed each $12 \mathrm{~h}$. Because of it, and despite Fig.1a, the experimental device is symmetrical: electrolyte reservoir supplies electrolytes to both collector compartments, and the EOF is collected from both collector compartments depending on the position of anode and cathode.

Soil samples were taken only at the beginning (before the soil was placed in the cell) and at the end of the experiments, but not during the experiments, in order to maintain the permeability of soil . At the end of the experiments (post-mortem analysis), soil samples were taken in four intermediate positions between the anode and cathode wells (position 1 is near anode and position 4 near cathode, according to the position at time zero; note again that anode and cathode electrodes changed their position due to the polarity reversal applied). In turn, each intermediate sampling point also considered four 
different samples in different axial positions. Additionally, daily samples of electrolyte were taken in the electrode compartments.

Oxyfluorfen and xylene concentrations, in soil and electrode wells, were measured by HPLC (Jasco, Japan) with UV detector. Acetonitrile/water, 70/30 by volume, was the mobile phase, and the column is a Kinetex $5 \mu \mathrm{m}$ Biphenyl $100 \AA$, 150 x $4.5 \mathrm{~mm}$ column (Phenomenex, USA) with an isocratic flow rate of $0.6 \mathrm{~mL} \mathrm{~min}{ }^{-1}$. The UV wavelength was $220 \mathrm{~nm}$ and $20 \mu \mathrm{L}$ of injection volume.

Soil moisture was calculated by measuring the loss weight after water evaporation at $105^{\circ} \mathrm{C}$ during 24 hours. $\mathrm{pH}$, electrical conductivity and nutrient concentrations (phosphate, nitrate and ammonium) in soil were measured as follows: $10 \mathrm{~g}$ of dried soil adding $25 \mathrm{~mL}$ of Milli-Q water were agitated during 30 minutes; then, it was left to settle for a few minutes and supernatant liquid was filtered with $0.2 \mathrm{~mm}$ nylon filters; A probe multiparameter (SENSLON, HACH) was used to measure the conductivity and $\mathrm{pH}$, and nutrients were measured with a photometer Gallery (Thermo Scientific). The active microbial concentration in the soil was expressed as Colony Forming Units (CFU) per gram of dry soil as reported by Ramírez et al., [12]. Samples were prepared following next procedure: $10 \mathrm{~mL}$ of saline solution $(0.9 \%)$ were added to $1 \mathrm{~g}$ of wet soil and the mixture was stirred for 3 min with a vortex agitator. Aliquots of supernatant $(100 \mu \mathrm{L})$ were taken and placed on Petri dishes containing Luria-Bertani growth medium (composition per liter of Milli-Q water: $10.0 \mathrm{~g} \mathrm{NaCl}, 5.0 \mathrm{~g}$ yeast extract and $10.0 \mathrm{~g}$ casein peptone, $15.0 \mathrm{~g}$ of European Bacteriological Agar and $10.0 \mathrm{~g}$ of glucose as carbon source). The plate's incubation time was $24 \mathrm{~h}$ at $26.5^{\circ} \mathrm{C}$. Temperature was measured in soil by thin temperature probes or thermocouples.

\section{Results and discussion}

The present work considers two possible real situations to achieve the electro- 
bioremediation of oxyfluorfen-polluted soil. The first one (Case 1) corresponds to a situation in which the polluted soil already contains inorganic nutrients and an autochthonous microbial population adapted to use the organic pollutant as the carbon source (a possible situation in historically polluted sites). Thus, the proposed treatment consists of using electrokinetics for the mobilization of pollutants, nutrients and microorganisms, in order to improve the contact between them (biostimulation), but trying to keep experimental conditions in suitable values for microbial life.

The second situation (case studies 2 and 3) corresponds to a condition in which the polluted soil does not contain microbial population nor nutrients (i.e., a recently polluted soil as consequence of recent spills). In that case, it is necessary to introduce microorganism and nutrients into the soil. The present work considers that a correct option to do it is through the inclusion of a biological barrier in the central position, far from the areas near the electrodes. Moreover, this option implies that pollutants should be mobilized with groundwater flow to pass through the barrier. The BB1 barrier is a conventional fixed bed biofilm reactor, which was previously and externally developed, while BB2 is just a mixture of soil and microbial suspension.

Fig. 2a shows the electro-osmotic flux (EOF) through the soil during the tests, which also indicates the water that leaves the system and it must be replaced. EOF in case 1 is much higher (approximately 5 times higher) than the observed in the other two experiments, while the difference between EOF in cases 2 and 3 is very low. These differences indicate that the water mobility and the mixing effects inside the soil (in both directions because of the polarity reversal) are much higher in case 1 . Fig. 2b shows the current density across the soil during the three experiments. Current density remained constant and the higher value was again observed in case 1, while the lower value corresponds to case 2 . 
Fig. 3 shows the average electrical conductivity values across the soil positions at the starting and the end of thetests. The figure shows the values at different soil intermediate points. The left part (vertical axis) of the Figure corresponds to the anode compartment, while the right vertical axis corresponds to the cathode compartment (at time zero). It can be observed that the average soil conductivity level is higher in experiment 1, as compared to the other two experiments, because the soil in experiment 1 contains the same electrolyte salt solution but also the BHB inorganic growth medium in every part of the soil. The water level control system (Fig. 1a) continuously replaced water and electrolytes.

Figures 2 and 3 thus show important differences between test 1 and the tests 2 and 3 . It is well known that EOF is directly proportional to the applied voltage gradient, to the zeta potential (that, in turn, depends on the ionic concentration) and the dielectric constant of the fluid, and inversely proportional to the fluid viscosity [4] and that EOF through low permeability regions is significantly greater that the EOF achieved in higher porosity regions. The introduction of the barriers in the present work caused a significant EOF decrease, which could be also related to the decrease in the current density and the soil conductivity. The EOF decrease, especially in test 1 , was expected as biobarriers increased porosity in the central position, and porosity can be one of the main factors that influences on it. Some contradiction can be found by comparing results in tests 2 and 3, as EOF is lower using $\mathrm{BB} 2$ but the $\mathrm{BB} 1$ porosity (made by gravel particles) is clearly higher. However, differences are very low. Therefore, it could be considered that other variables such as ionic concentration (directly related to current density and soil conductivity) are also important and they could influence the BB2 experiment performance, causing the lower EOF value. It must be noted that variables such as voltage gradient, fluid dielectric constant and viscosity remained 
constant in all tests.

Some authors have previously studied the influence of the insertion of a permeable reactive barrier (PRB) in electrokinetic remediation process, and different results have been reported. Wang et al. [13] reported that a Pd/Fe PRB inclusion caused that EOF decreased 1.8 times in an electrokinetic remediation process of hexaclorobenzenepolluted soil. Kebria et al. [14] reported similar results. On the contrary, the same authors [15] reported that a $\mathrm{Cu} / \mathrm{Fe} \mathrm{PRB}$ insertion caused that EOF increased, again in an electrokinetic remediation process of hexaclorobenzene-polluted soil. Regarding the application of biological PRB (biobarriers), the authors of the present work previously reported results that compared the performance of $\mathrm{BB} 1$ and $\mathrm{BB} 2$ in the electrokinetic remediation of diesel hydrocarbons polluted clay soil, and they found that EOF was higher when using BB1 [9] and that the EOF was slightly higher if no barrier was used [10].

Fig. 4 shows average values of soil $\mathrm{pH}$ and temperature at the starting and the end of the tests. It can be observed that both conditions were nearly identical in the three cases, although a slight temperature increase was observed when using biobarriers (experiments 2 and 3) and it could be related to a higher electrical resistance associated to the barrier because a current density decrease was also observed. The temperature and the $\mathrm{pH}$ profiles were homogeneous in every part of the soil and no gradients were observed. According to previous results [10], polarity reversal allowed an accurate control of the experimental conditions, especially regarding $\mathrm{pH}$. Different strategies have been proposed to maintain a suitable $\mathrm{pH}$ in the soil during electro-bioremediation processes [16]. One of the most interesting methods is the periodic change of the polarity of the electric field (so-called as periodic polarity reversal). Different works have been reported in the last decade regarding the application of polarity reversal in order to control $\mathrm{pH}$ 
during electro-bioremediation treatment, and some of them also showed beneficial effects in temperature and moisture control [17].

Thus, it can be considered that such conditions are adequate and identical in the three experiments.

Fig. 5 shows the average values of the microorganism's concentration in the different parts of the soil at the beginning (only experiment 1 , continuous line) and the end (dashed line) of the tests. It can be observed that the biological activity in case 1 remains in every part of the soil after two weeks. In the cases 2 and 3, it could be expected that the biological activity was only observed in areas near the central biobarrier, but the results after two weeks show again that microorganims are distributed in every portion of the soil and thus, microbial mobility in both directions may have happened. Similar results have been reported using similar barriers [9]. The presence of microorganisms in zones outside from the central biobarrier is supposed to be mainly caused from the biofilm detachment process and the EK transport of the microorganisms. The biofilm detachment process resulted from biomass growth and the movement of water through the biobarrier. Regarding the EK transport of microorganisms in the soil, it has been extensively reported that it can be produced both by electrophoresis and electro-osmosis [18]. Note that polarity reversal changes the direction of both mechanisms in the present work every 12 h. It was not observed a decrease in microbial concentration near the electrodes, indicating again a successful $\mathrm{pH}$ control. It can be observed also in Fig. 5 that the average concentration values are very similar in all tests.

Fig. $6(a, b, c)$ shows the average values of oxyfluorfen concentration in the soil at the beginning and the end of the tests. No oxyfluorfen was detected in the electrode compartments. Fig. 6d shows the oxyfluorfen removal efficiencies after two weeks. In addition, the efficiency results (negligible values) in the two reference tests (No EK and 
No Bio tests) have been included in Fig.6d. It can be observed that the oxyfluorfen removal efficiency after only two weeks is still low, $15 \%$ is the best result, while higher removal efficiencies have been previously obtained when using the same technology to remediate hydrocarbon-polluted soil [10]. It is considered that the low efficiency observed can be explained because oxyfluorfen is a non-polar pollutant which is distributed in the form of charged micelles, more difficult to be transported under the application of electrokinetics than ions. Moreover, according to its chemical characteristics, oxyfluorfen is strongly adsorbed to most soils, especially soils with high organic matter and clay percentages [19] and thus it should have a very low mobility in soil. Halogenated organic compounds are mobilized mainly by the contribution of electroosmosis and they need the help of solvents or surfactants [4]. It was observed in our experiments that xylene (the main solvent included in the commercial pesticide used in this work) was evaporated during the first week and it could help to explain the reduction in the mobilization of oxyfluorfen. However, it has been recently reported by the same authors [20] that the EK-biostimulation option for oxyfluorfen removal could reach efficiencies of about $40 \%$ after 11 weeks. Additionally, the application of surfactants in the electrode compartments could improve the oxyfluorfen removal efficiency as recently reported [21]. The present work used just 2 weeks because it is the experimentation time that allowed us to understand the differences between the three strategies under study.

Thus, taking into account the results of the two reference tests, it seems that the combination of bioremediation and electrokinetics allowed a little advance in the remediation, while the separate options (only biological or only electrokinetics) alone obtained negligible oxyfluorfen removal efficiencies. It can be observed that the best option in the present work is the first one (case 1) while biobarriers caused a decrease in the removal efficiency, considering the retention time 2 weeks. This result could be 
directly related to the decrease in EOF when using the barriers as the experimental conditions related to the biological activity (temperature, $\mathrm{pH}$ and microorganisms concentration) are nearly identical in all experiments, and also nutrients are always available in excess concentrations. Regarding the EOF decrease, it could be explained because of the higher porosity in the biobarrier zones which also results in lower values of current density in tests 2 and 3 . In addition, the lower ionic conductivity as compared to case 1 (because the BHB nutrient medium was not included in the whole soil) can also help to explain the observations.

Authors consider that further studies are necessary to improve the remediation efficiency results (i.e. using higher retention times or improving the pollutant mobility). Additionally, full scale electro-bioremediation studies are scarce and they are considered as one of the main future challenges and research needs. Recently, Barba et al. [22] reported a first study about the application of biobarriers in the electrobioremediation of oxyfluorfen polluted soil. Results were not successful as it was difficult to maintain adequate environmental conditions (temperature, $\mathrm{pH}$ and nutrients availability) that allowed microbial activity. Because of that, additional efforts regarding full scale studies should be considered in the future.

\section{Conclusions}

The EK-biostimulation option obtained $15 \%$ of oxyfluorfen removal after two weeks. The EK-bioaugmentation option (biobarrier inclusion) caused a slight decrease in removal efficiency because a decrease in the EOF. Despite the low efficiencies obtained, biobarriers could be considered as a valuable option to improve the EK remediation of oxyfluorfen-polluted soil, because they allowed the introduction of microorganisms and nutrients into the soil, and thus it would allow the in-situ pollutant biodegradation. The biological activity was not only located in the central biobarrier 
zone but it also reached the whole soil positions. The results suggest that the removal efficiencies were still low after two weeks because oxyfluorfen mobility was low and higher treatment times are recommended.

\section{Acknowledgements}

Financial support from the Spanish Ministry of Economy, Industry and Competitiveness and European Union through project CTM2016-76197-R (AEI/FEDER, UE) is

gratefully acknowledged. The FPI doctoral grant to Ms. Silvia Barba BES-2014-069662 is also acknowledged.

Conflicts of interest: none.

\section{References}

[1] M.A. Rodrigo, M. Oturan, M.A. Oturan, Electrochemically Assisted Remediation of Pesticides in Soils and Water: A Review, Chem. Rev. 114 (2014) 8720-8745.

[2] H. Zhao, J. Xu, F. Dong, X. Liu, Y. Wu, X. Wu, Y. Zheng, Characterization of a novel oxyfluorfen-degrading bacterial strain Chryseobacterium aquifrigidense and its biochemical degradation pathway, App. Microbiol. Biotechnol. 100 (2016) $6837-6845$.

[3] T. Castelo-Grande, P.A. Augusto, P. Monteiro, A.M. Estevez, D. Barbosa, Remediation of soils contaminated with pesticides: A review, Int. J. Environ. Anal. Chem. 90 (2010), 438-467.

[4] K.R. Reddy, C. Cameselle, Overview of Electrochemical Remediation Technologies, in: K. R. Reddy, C. Cameselle, (Eds.), Electrochemical Remediation Technologies for Polluted Soils, Sediments and Groundwater. Eds. John Wiley \& Sons, Inc., Hoboken, New Jersey, 2009, pp. 3-29. 
[5] H.I. Gomes, C. Dias-Ferrerira, A.B. Ribeiro, Electrokinetic remediation of organochlorines in soil: Enhancement techniques and integration with other remediation technologies, Chemosphere 87 (2012) 1077-1090.

[6] L.Y. Wick, L. Shi, H. Harms, Electro-bioremediation of hydrophobic organic soilcontaminates: A review of fundamental interactions, Electrochim. Acta 52 (2007) 3441-3448.

[7] R.T. Gill, M.J. Harbottle, J.W.N. Smith, S.F. Thornton, Electrokinetic-enhanced bioremediation of organic contaminants: A review of processes and environmental applications, Chemosphere 107 (2014) 31-42.

[8] C.M. Kao, S.C. Chen, J.K. Liu, Development of a biobarrier for the remediation of PCE-contaminated aquifer, Chemosphere 43 (2001) 1071-1078.

[9] E. Mena, J. Villaseñor, M.A. Rodrigo, P. Cañizares, Influence of electric field on the remediation of polluted soil using a biobarrier assisted electro-bioremediation process, Electrochim. Acta 190, (2016) 294-304.

[10] E.M. Ramírez, J.V. Camacho, M.A. R. Rodrigo, P.C. Cañizares, Combination of bioremediation and electrokinetics for the in-situ treatment of diesel polluted soil: a comparison of strategies, Sci. Total Environ. 533 (2015)307-316.

[11] E. Moliterni, R.G. Jiménez-Tusset, M. Villar Rayo, L. Rodríguez, F.J. Fernández, J. Villaseñor, Kinetics of biodegradation of diesel fuel by enriched microbial consortia from polluted soils, Int. J. Environ. Sci. Tech. 9 (2012) 749-758.

[12] E.M. Ramírez, C.S. Jiménez, J.V. Camacho, M.A.R. Rodrigo, P.C. Cañizares, Feasibility of coupling permeable bio-barriers and electrokinetics for the treatment of diesel hydrocarbons polluted soils, Electrochim. Acta, 181 (2015) 192-199.

[13] J. Wang, Z. Li, X. Lu, S. Yuan, Remediation of a hexaclorobenzene contaminated 
soil by surfactant-enhanced electrokinetics coupled with microscale Pd/Fe PRB, J. Hazard. Mater. 184 (2010) 184-190.

[14] D.Y. Kebria, M. Taghizadeh, J.V. Camacho, N. Latifi, Remediation of PCE contaminated clay soil by coupling electrokinetics with zero-valent iron permeable reacive barrier, Environ. Earth Sci. 75 (2016) 699-709.

[15] J. Wang, Z. Li, S. Yuan, X. Lu, Removal of hexaclorobenzene from kaolin by electrokinetics coupled with $\mathrm{Cu} / \mathrm{Fe}$ PRB, J. Hazard. Toxic Radioact. Waste, 15 (2011) 175-179.

[16] A.T. Yeung, Y.Y. Gu, A review on techniques to enhance electrochemical remediation of contaminated soils, J. Hazard. Mater. 195 (2011) 11-29.

[17] T. Li, S. Guo, B. Wu, L. Zhang, Y. Gao, Effect of polarity-reversal and electrical intensity on the oil removal from soil, J. Chem. Technol. Biotechnol. 90 (2015) 441-448.

[18] M.F. Deflaun, C.W. Condee, Electrokinetic transport of bacteria, J. Hazard. Mater. 55 (1997) 263-277.

[19] USEPA. United States Environmental Protection Agency. Reregistration eligibility decision (RED), oxyfluorfen; 2002 [EPA: 738-R-02-014].

[20] S. Barba, J. Villaseñor, M.A. Rodrigo, P. Cañizares, Can electro-bioremediation of polluted soils perform as a selfsustainable process?, J. Appl. Electrochem., 48 (2018) 579-588.

[21] S. Barba, M. Carvela, J. Villaseñor, M.A. Rodrigo, P. Cañizares, Improvement of the electro-bioremediation process of a non-polar herbicide polluted soil by means of surfactant addition, Sci. Total Environ. 650 (2019) 1961-1968. 
[22] S. Barba, R. López-Vizcaíno, C. Sáez, J. Villaseñor, P. Cañizares, V. Navarro, M.A. Rodrigo, Electro-bioremediation at the prototype scale: What it should be learned for the scale-up, Chem. Eng. J., 334 (2018) 2030-2038. 